\title{
A Standardized Framework
}

for Fluorescence-Guided Margin Assessment for Head and Neck Cancer Using a Tumor Acidosis Sensitive Optical Imaging Agent

Pieter Jan Steinkamp $\odot,{ }^{1}$ Floris Jan Voskuil, ${ }^{2,3}$ Bert van der Vegt, ${ }^{3}$ Jan Johannes Doff, ${ }^{3}$ Kees-Pieter Schepman, ${ }^{2}$ Sebastiaan Antonius Hendrik Johannes de Visscher, ${ }^{2}$ Wendy Kelder, ${ }^{4}$ Yalia Jayalakshmi, ${ }^{5}$ Jinming Gao, ${ }^{5,6}$ Baran Devrim Sumer, ${ }^{5,7}$ Gooitzen Michell van Dam, ${ }^{1,8}$ Max Johannes Hendrikus Witjes ${ }^{2}$

\footnotetext{
${ }^{1}$ Department of Surgery, Nuclear Medicine and Molecular Imaging, University of Groningen, University Medical Center Groningen, PO Box 30.0019700, RBGroningen, The Netherlands

${ }^{2}$ Department of Oral \& Maxillofacial Surgery, University of Groningen, University Medical Center Groningen, PO Box 30.0019700, RBGroningen, The Netherlands

${ }^{3}$ Department of Pathology \& Medical Biology, University of Groningen, University Medical Center Groningen, PO Box 30.0019700, RBGroningen, The Netherlands

${ }^{4}$ Department of Surgery, Martini Ziekenhuis, Groningen, The Netherlands

${ }^{5}$ OncoNano Medicine Inc., Dallas, TX, USA

${ }^{6}$ Department of Pharmacology, University of Texas Southwestern Medical Center, Dallas, TX, USA

${ }^{7}$ Department of Otolaryngology Head and Neck Surgery, Simmons Comprehensive Cancer Center, University of Texas Southwestern Medical Center, Dallas, TX, USA

${ }^{8}$ Tracer Europe B.V./AxelaRx B.V., Groningen, The Netherlands
}

\begin{abstract}
Purpose: Intra-operative management of the surgical margin in patients diagnosed with head and neck squamous cell carcinoma (HNSCC) remains challenging as surgeons still have to rely on visual and tactile information. Fluorescence-guided surgery using tumor-specific imaging agents can assist in clinical decision-making. However, a standardized imaging methodology is lacking. In this study, we determined whether a standardized, specimen-driven, fluorescence imaging framework using ONM-100 could assist in clinical decision-making during surgery.

Procedures: Thirteen patients with histologically proven HNSCC were included in this clinical study and received ONM-100 $24 \pm 8 \mathrm{~h}$ before surgery. Fluorescence images of the excised surgical specimen and of the surgical cavity were analyzed. A fluorescent lesion with a tumor-to-background ratio (TBR) $>1.5$ was considered fluorescence-positive and correlated to standard of care (SOC) histopathology.

Results: All six tumor-positive surgical margins were detected immediately after excision using fluorescence-guided intra-operative imaging. Postoperative analysis showed a median TBR $( \pm \mathrm{IQR})$ of the fluorescent lesions on the resection margin of $3.36 \pm 1.62$. Three fluorescence-
\end{abstract}

\footnotetext{
Pieter Jan Steinkamp and Floris Jan Voskuil contributed equally to this work.Pieter Jan Steinkamp and Floris Jan Voskuil share first authorship.

Correspondence to: Max Witjes; e-mail: m.j.h.witjes@umcg.nl
} 
positive lesions in the surgical cavity were biopsied and showed occult carcinoma and severe dysplasia, and a false-positive fluorescence lesion.

Conclusion: Our specimen-driven fluorescence framework using a novel, $\mathrm{pH}$-activatable, fluorescent imaging agent could assist in reliable and real-time adequate clinical decisionmaking showing that a fluorescent lesion on the surgical specimen with a TBR of 1.5 is correlated to a tumor-positive resection margin. The binary mechanism of ONM-100 allows for a sharp tumor delineation in all patients, giving the surgeon a clinical tool for real-time margin assessment, with a high sensitivity.

Key words: Fluorescence-guided surgery, $\mathrm{pH}$ activation, Tumor type-agnostic imaging, Margin assessment, Standardization

\section{Introduction}

Optimal surgical management of head and neck squamous cell carcinoma (HNSCC) requires a tumor resection with adequate surgical margins as inadequate surgical margins are associated with an increased chance of local recurrence and metastasis, eventually causing reduced overall survival [1$3]$. Fluorescence-guided surgery (FGS) is a novel imaging technique that allows tumor visualization during surgery by targeting tumor-specific biomarkers, proteins, or receptors. Multiple clinical phase 1 and phase 2 studies have investigated a variety of fluorescent imaging agents to enhance tumor detection, showing the potential of tumortargeted FGS to guide surgical decision-making in a variety of clinical settings $[4,5]$. However, the clinical translation of fluorescent-guided margin assessment lacks a standardized imaging method that allows immediate and reliable feedback for the surgeon. In this study, we provide an ex vivo specimen-driven imaging methodology in patients with HNSCC by implementing a promising $\mathrm{pH}$ dependent, tumor type-agnostic imaging agent called ONM-100 [6]. We suggest that ex vivo imaging might be preferable over in vivo imaging since external factors influencing imaging results are limited. Subsequently, we hypothesize the potential clinical effects of this standardized method for our patient cohort.

In recent years, a variety of tumor-specific fluorescence optical imaging agents have been developed. Different targeting strategies, like fluorophores conjugated to monoclonal antibodies (MoAb) or PARP1 inhibitors, have been clinically evaluated in HNSCC [5, 7-9]. Despite interesting results, target heterogeneity within cancer and target expression on the majority of healthy cells might cause falsepositive signal and a limited discriminative strength [10]. Moreover, as the half-life of a MoAb is on average 7-10 days, administration needs to be done 2-5 days prior to surgery, which comes with logistical challenges $[5,11]$. The optical imaging agent ONM-100, consisting of micelles containing indocyanine green (ICG), can overcome these limitations by targeting more ubiquitous tumor characteristics, namely tumor acidosis [12]. The mechanism of action of the imaging agent is described previously [12]. Briefly, the micelles dissociate in the acidic tumor microenvironment, allowing the ICG to un-quench and become fluorescent. As non-tumor tissue has a physiological $\mathrm{pH}$, no "background" fluorescence activation is observed in nontumor tissue, resulting in high tumor-to-background ratios (TBRs). The working mechanism has recently been clinically evaluated in 30 patients, showcasing the applicability of ONM-100 in four different tumor types [6].

As the field of FGS is rapidly expanding, data collection and implementation of fluorescence-guided surgery into the clinical workflow needs to be standardized, since results are highly affected by factors other than the distribution of the fluorophore itself. Therefore, we validate a novel framework using a $\mathrm{pH}$-activatable optical imaging agent in HNSCC (Supplemental Figure S1). We hypothesize that our standardized framework with a specimen-driven approach, combined with the next-generation $\mathrm{pH}$-activatable optical imaging agent, allows for reliable real-time intra-operative decision-making. In this study, we present data of 13 HNSCC patients who received ONM-100 $24 \mathrm{~h}$ prior to surgery. Our data support the potential clinical value of this standardized framework using ONM-100 to alter real-time clinical decision-making, since all tumor-positive surgical margins were detected immediately after excision in the ex vivo environment.

\section{Materials and Methods}

\section{Study Design}

This study was conducted at the University Medical Center Groningen (UMCG) and data was analyzed retrospectively. The clinical study was approved by the Institutional Review Board (IRB number 2017/580) of the UMCG. Patients with histopathologically proven head and neck squamous cell carcinoma (HNSCC) scheduled for surgical removal were enrolled as part of a larger clinical trial (the Netherlands National Trial Register 7085, EudraCT number 2017003543-38) assessing the safety and generic applicability and optimal dose of ONM-100 [6]. All patients gave their informed consent after being informed about the study. In the current study, we investigated if ONM-100 was suitable 
for intra-operative margin assessment in HNSCC surgery using a standardized fluorescence workflow (Supplemental Figure S2).

\section{ONM-100 Conjugation}

ONM-100 consists of polymeric micelles labeled with indocyanine green (ICG), as described previously [12]. ONM-100 was administered intravenously $24 \mathrm{~h} \pm 8 \mathrm{~h}$ before surgery in different dosages, which has been extensively described in our previous study [6].

\section{Standardized Method for Fluorescence-Guided Clinical Decision-Making}

\section{Specimen-Driven Margin Assessment}

The excised surgical specimen of all patients was retrospectively analyzed using the PEARL-trilogy ${ }^{\circledR}$ imaging device (Li-COR BioSciences Inc., Lincoln, NE, USA). This closed-field device is suitable for ex vivo tissue imaging, as it eliminates ambient light and enables standardization of imaging between specimens. The tumor and all resection planes of the specimen were imaged immediately $(<10 \mathrm{~min})$ after tumor excision. The tissue thickness varied in size up to $10 \mathrm{~cm}$. Imaging resolution was $85 \mu \mathrm{m}$. If a fluorescent lesion was detected at one of the resection planes, this lesion was manually delineated and considered fluorescencepositive if a TBR of $>1.5$ was obtained. This was based on an earlier reported study which determined 1.5 as adequate for discrimination between tumor and non-tumor tissue in FGS [13]. The fluorescent lesion was macroscopically delineated and the remaining, non-fluorescent, area of the respective resection plane was considered as background to determine the mean fluorescence intensity (MFI). For each patient, MFI lesion/MFI background was used to determine the TBR on the resection planes. If a fluorescent lesion was detected, the location of the fluorescent lesion was correlated based on anatomical information with fluorescence images of the surgical cavity. For orientation of the surgical specimen, sutures were applied on the specimen to allow for maximal orientation, as per standard of care. For one patient, the specimen was too large for the sample stage of the PEARL-trilogy ${ }^{\circledR}$ imaging device. For this patient, the SurgVision Explorer Air ${ }^{\circledR}$ Vault was used (details described below).

\section{Surgical Cavity Driven Margin Assessment}

For the detection of remaining tumor in the surgical cavity, fluorescence imaging using two intra-operative camera devices, the SurgVision Explorer Air ${ }^{\circledR}$ (SurgVision B.V., Groningen, the Netherlands) and the NovaDaq Spy Elite (Stryker, Kalamazoo, MI, USA), was performed in vivo. All additional fluorescent lesions detected prior to surgery or after surgery in the surgical cavity were biopsied, based on the definition of
TBR $>1.5$, and analyzed for final histopathological examination if deemed safe and feasible by the attending surgeon. In vivo imaging took approximately 5-10 min per procedure.

\section{Ex vivo Correlation of Intra-operative Fluores- cence Results}

To validate fluorescence imaging results, fluorescence results were correlated with final histopathology. Following SOC, the surgical specimen was formalin-fixed for at least $24 \mathrm{~h}$ and subsequently sliced in approximately $0.5 \mathrm{~cm}$ thick tissue slices. In the case a fluorescent lesion was detected on the surgical margin during imaging of the excised specimen, this was precisely correlated to the representative tissue slice. Next, tissue slices were embedded into formalin fixed paraffin embedded (FFPE) blocks according to SOC by a pathologist blinded for fluorescence results. Fluorescent areas at the tissue slices that were not initially selected for histopathological analysis by the blinded pathologist were additionally embedded. Histopathological assessment on hematoxylin and eosin (H\&E) stained $3 \mu \mathrm{m}$ tissue sections was done by a board-certified pathologist, blinded for fluorescence, and according to SOC.

\section{Fluorescence Imaging Devices}

In vivo fluorescence images were obtained using two intraoperative cameras, namely the Explorer Air ${ }^{\circledR}$ (SurgVision B.V., Groningen, the Netherlands) or the Novadaq SPY Elite ${ }^{\circledR}$ (Stryker, Kalamazoo, MI, USA). Fluorescence imaging with the SurgVision Explorer Air ${ }^{\circledR}$ was performed as previously described by our group [5]. The Novadaq Spy Elite contains a light emitting diode (LED) with an excitation wavelength of $805 \mathrm{~nm}$, designed for the detection of ICG. White light images and fluorescence videos were obtained during surgery. The working distance above the surgical field was $30 \mathrm{~cm}$. Fluorescence gain could be manually adapted during imaging.

The excised whole specimen and tissue slices were imaged using the PEARL-trilogy ${ }^{\circledR}$ imaging device, using a CCD camera in the NIR wavelength (peak exCitation 785 $\mathrm{nm}$, peak emission $820 \mathrm{~nm}$ ). The field of view of $11.2 \mathrm{~cm} \times$ $8.4 \mathrm{~cm}$ and the focus point can be adjusted based on specimen height. All images were stored in TIFF format and post-processed by applying a color scheme for the benefit of fluorescence signal intensity visualization and analyzed using Fiji (version 2.0.0-rc-68/1.52h). The fluorescence values did not change by applying the color scheme.

\section{Results}

Thirteen patients with histopathologically proven HNSCC were included. Patient and fluorescence imaging characteristics are depicted in Table 1. 
Table 1. Patient characteristics

\begin{tabular}{|c|c|c|c|c|c|c|c|}
\hline Patient number & Age & Location of tumor & Tumor stage & Margin status & Distance to closest margin & $\begin{array}{l}\text { Whole specimen } \\
\text { fluorescence }\end{array}$ & $\begin{array}{l}\text { Surgical cavity } \\
\text { fluorescence }\end{array}$ \\
\hline Patient 01 & 50 & Mandible & pT4N1M0 & Close & $3.5 \mathrm{~mm}$ & - & - \\
\hline Patient 02 & 69 & Floor of mouth & pT3N2bM0 & Positive & Cut through & + & - \\
\hline Patient 03 & 53 & Tongue & pT3N0Mx & Close & $3 \mathrm{~mm}$ & + & + \\
\hline Patient 04 & 46 & Floor of mouth & pT3N0Mx & Positive & $<1 \mathrm{~mm}$ & + & - \\
\hline Patient 05 & 80 & Cheek & pT1N0Mx & Positive & Cut through & + & - \\
\hline Patient 06 & 79 & Cheek & pT2N0Mx & Close & $3 \mathrm{~mm}$ & - & - \\
\hline Patient 07 & 70 & Tongue & pT3N2bMx & Positive & $<1 \mathrm{~mm}$ & + & + \\
\hline Patient 08 & 48 & Floor of mouth & pT1N1Mx & Free & $>5 \mathrm{~mm}$ & + & - \\
\hline Patient 09 & 69 & Mandible & pT2N0Mx & Positive & $<1 \mathrm{~mm}$ & + & + \\
\hline Patient 10 & 85 & Mandible & pT4N0Mx & Positive & Cut through & + & + \\
\hline Patient 11 & 84 & Palate & pT1N0Mx & Close & $3 \mathrm{~mm}$ & + & - \\
\hline Patient 12 & 58 & Tongue & pT1N0Mx & Close & $2 \mathrm{~mm}$ & + & - \\
\hline Patient 13 & 77 & Tongue & pT2N0Mx & Free & $>5 \mathrm{~mm}$ & - & - \\
\hline
\end{tabular}

Whole specimen fluorescence: presence of a fluorescent lesion at the edge of the resected specimen. Surgical cavity fluorescence: presence of a fluorescent lesion in the surgical cavity during surgical excision. $+=$ positive for fluorescence, $-=$ negative for fluorescence

\section{Ex vivo Specimen-Driven Margin Assessment}

Both the mucosal and the deep resection planes were evaluated by fluorescence imaging of the excised specimen $(N=13$, Figs. 1 and 2). All six histopathologically proven tumor-positive surgical margins (viable tumor cells $\leq 1 \mathrm{~mm}$ of margin) were detected using our standardized ex vivo fluorescence imaging framework, which enables accessible and fast identification of sharply delineated fluorescent signals $(6 / 6,100 \%$ sensitivity). Five out of these six tumor-positive surgical margins were located at the deep resection margin (Supplemental Figure S2). All fluorescent lesions correlated to the location of the histopathologically tumor-positive surgical margin with a median TBR of $3.36 \pm$ 1.62 (Supplemental Figure S3). Additionally, five histopathologically proven close margins (viable tumor cells $1-5 \mathrm{~mm}$ of margin) were diagnosed in this study, of which three were determined as fluorescence-positive. Two were scored as fluorescence-negative, which had both a surgical margin $\geq$ $2 \mathrm{~mm}$ (Table 1). Important to state, all surgical margins that were assessed as fluorescence-negative had a surgical margin $>2 \mathrm{~mm}$. Of the two specimens that were histopathologically diagnosed with tumor-negative surgical margins (>5 mm), one specimen was scored as fluorescence-positive at one of the resection margins, which was found to be caused by localized fluorescence around the salivary glands. This particular case showed no elevated fluorescence signal in the surgical cavity (Table 1). Fluorescence of the salivary glands was only observed in this single patient.

\section{Surgical Cavity Driven Margin Assessment}

During fluorescence imaging of the surgical cavity and peripheral surfaces in HNSCC $(N=13)$, four fluorescent lesions could be identified of which in three cases, a fluorescence-guided biopsy was performed. Three lesions were considered to be true positive. The mean TBR of these true positive biopsies was 4.68 (range 2.2-6.2) (Fig. 3). Patient 7 underwent tumor excision of a HNSCC located at the tongue/floor of the mouth. After initial tumor excision, a fluorescent lesion was detected in the surgical cavity (TBR 2.2 Fig. 3, panels a-c). Histopathological analysis of the biopsy showed squamous cell carcinoma; however, there was no direct correlation with the location of the tumor-positive resection margin at the excised specimen. The tumor-positive biopsy is therefore diagnosed as in-transit metastasis. Despite adjuvant radiotherapy (cumulative dose $70 \mathrm{~Gy}$ ) without chemotherapy due to severe comorbidity, the patient developed a recurrent tumor during radiotherapy located at the deep tongue musculature. During tumor resection of patient 9 , fluorescence imaging of the medial side of the surgical area showed a fluorescencepositive lesion (TBR 6.2, Fig. 3 panels $\mathrm{d}-\mathrm{f}$ ). The biopsy of this lesion showed severe dysplasia without invasive carcinoma. Histopathological analysis of the surgical specimen revealed a tumor-positive surgical margin at the medial resection plane along with the presence of high-grade dysplasia, which correlated with the location of the fluorescent lesion in the surgical cavity. Lastly, the surgical cavity of patient 10 showed a large area of fluorescence (TBR 5.7, panels g-i). Based on clinical arguments, related to the extent of the surgical defect, no biopsy was performed. The ex vivo fluorescence correlated with a focal tumor-positive surgical margin which corresponded to the location of the fluorescence in the surgical cavity (Supplemental Figure S2, panels q-t). In one case (patient 3), a falsepositive fluorescence signal was observed in the surgical cavity (Supplemental Figure S4). Here, the lesion was suspected for perineural growth; however, the histopathology showed no tumor involvement nor any other reason for a specific ONM-100 activation.

\section{Real-Time Surgical Margin Determination - Defining the Room for Improvement}

Three patients (patients 9, 11, 12) underwent an additional re-resection within a month after initial surgery according to SOC due to a tumor-positive or close surgical margin. During initial surgery of all three cases, the excised specimen was diagnosed as fluorescence-positive. 


\section{Realtime surgical specimen analysis}

\section{Mucosal resection margin}

a

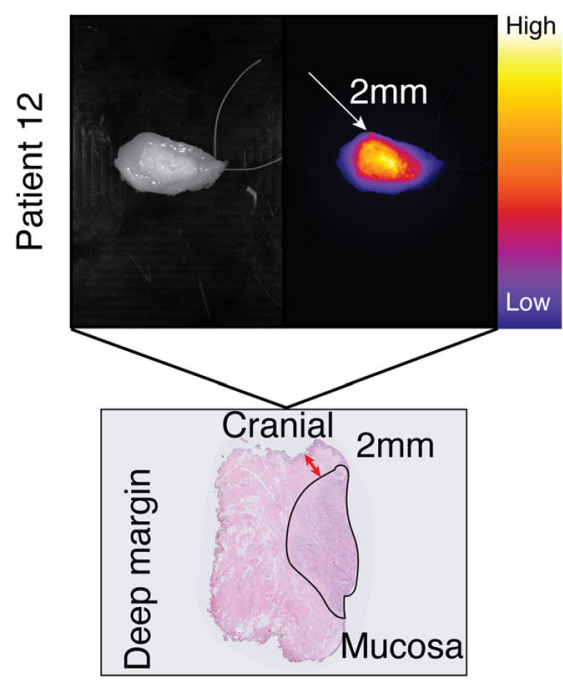

b

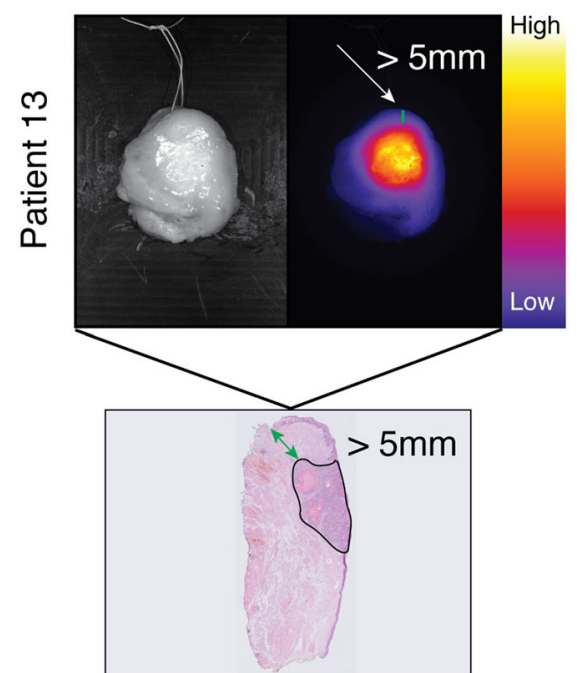

Deep resection margin

C
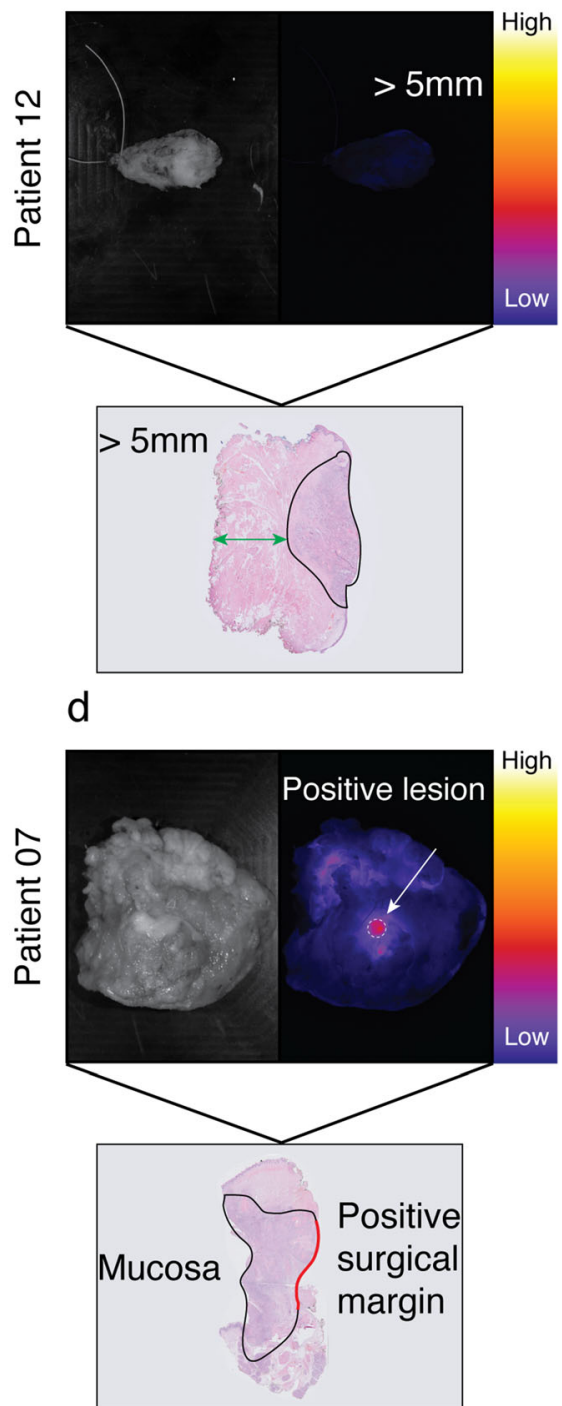

Fig. 1. Real-time surgical specimen analysis. Fluorescence-guided analysis of the mucosal tumor and of the deep resection margins in HNSCC patients was performed using Li-COR PEARL Trilogy. a Representative image of a mucosal tongue tumor with an insufficient superficial surgical margin $(2 \mathrm{~mm})$. b Representative image of a mucosal tongue tumor with a sufficient superficial surgical margin $(>5 \mathrm{~mm})$. c Representative image of a deep surgical resection margin negative for fluorescence which correlated with a tumor-negative margin. $\mathbf{d}$ Representative image of a positive fluorescent lesion on a deep resection margin correlating with a tumor-positive surgical margin.

Interestingly, during fluorescence imaging of the surgical cavity, no suspected lesions were detected in two of these cases (patients 11 and 12). This is congruent with the histopathological findings, showing a tumor-positive surgical margin $(<1 \mathrm{~mm})$, but no cut through of the tumor, thus tumor cells within $0-1 \mathrm{~mm}$ of the border. As a result, histopathological analyses after the surgical re-resection in a second surgery showed no remaining tumor cells in these two HNSCC (re-)resected specimens. The remaining patients diagnosed with a tumor-positive or close resection margin positive for fluorescence (patients $2,3,4,5,7,10$ ) received no additional surgery based on clinical considerations such as severe comorbidity as decided within the multidisciplinary tumor board.

\section{Discussion}

The field of fluorescence-guided surgery in oncology is expanding rapidly, but standardized methods to evaluate imaging results are not widely used. Our results indicate that 


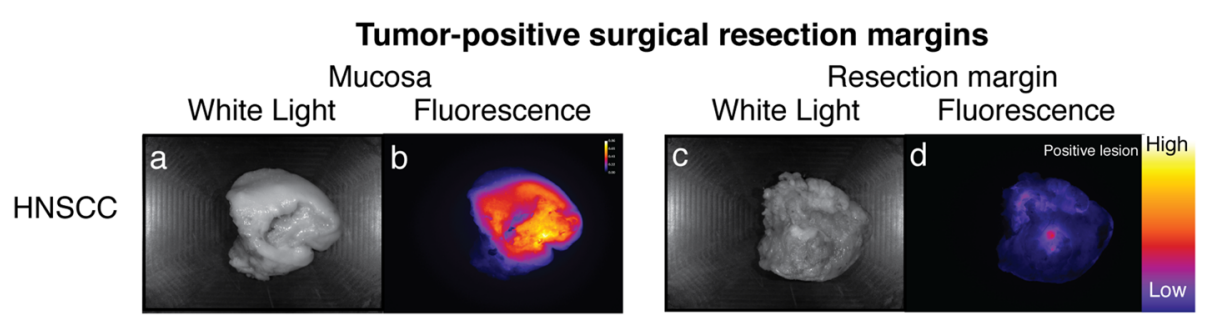

Fig. 2. Tumor-positive surgical margins. Representative images of an excided tumor specimen, visualized on the mucosal site (a-b) which shows clear delineation of the tumor by fluorescence imaging. Next, the deep resection margin (i.e., basal surface) is shown (c-d), illustrating a sharply delineated fluorescence-positive lesion which correlated with a tumor-positive surgical margin of the respective specimen.

a standardized ex vivo fluorescence-guided imaging method for margin assessment using the $\mathrm{pH}$-activated imaging agent ONM-100 shows substantial clinical potential for real-time intra-operative decision-making. We identified all tumorpositive margins by detecting fluorescent lesions at the surgical resection margin with a tumor-to-background ratio $>1.5$. This easy implementable framework, which can be implemented in the surgical theatre, can be used immediately after surgical excision allowing an alteration of the surgical strategy during initial surgery. Subsequently, we showed that ONM-100 can detect remaining tumor and severe dysplasia in the surgical cavity which was otherwise missed during standard of care surgery.

We suggest the use of our standardized fluorescence framework, combined with ONM-100, for real-time surgical margin assessment in HNSCC and surgery (Fig. 4). Briefly, when a fluorescent lesion at one of the resection margins is detected (threshold TBR > 1.5), and if anatomical borders allow an additional resection in situ, an immediate re- resection can be considered [13]. This imaging procedure can be performed in the surgical theatre to improve surgical outcome. After tumor excision, imaging of the surgical cavity is performed subsequently to fluorescence imaging of the freshly excised surgical specimen which can guide the surgeon in performing an immediate re-resection. Additionally, fluorescence imaging of the surgical cavity can detect occult tumor and/or dysplastic lesions, whether or not related to the primary tumor, as highlighted by the observations in our cohort, in which one occult additional tumor lesion and a dysplastic lesion were found using fluorescence imaging of the surgical cavity. We show that FGS using ONM-100 could guide the surgeon to perform an immediate reresection and potentially prevent (unnecessary) additional re-resections or adjuvant treatment. This potential is underlined by the fact that after an initial tumor-positive surgical margin, two HNSCC re-resections in this series were negative for residual tumor. In the unfortunate event that it is not possible to perform an additional resection, which

\section{Realtime in vivo analysis}

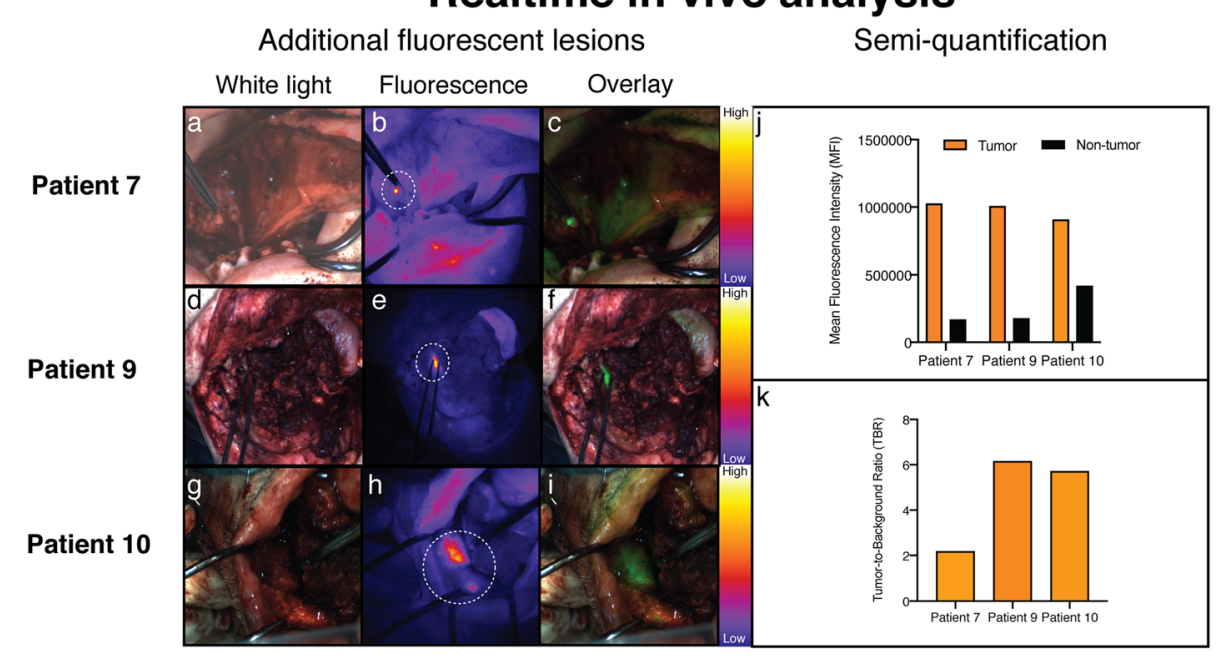

Fig. 3. Real-time in vivo HNSCC analysis. Patient 7 with an in-transit metastasis detected after biopsy of a fluorescent spot in the surgical cavity $(\mathbf{a}-\mathbf{c})$. Patient 9 with a tumor in the mandible with a positive spot for fluorescence in the surgical cavity, which showed high-grade dysplasia without invasive carcinoma (d-f). Patient 10 with a tumor in the mandible with a clear fluorescent spot indicating a focal tumor-positive surgical margin as confirmed by histopathological assessment (g-i). Mean fluorescence intensity (MFI) of the fluorescent spot compared to the background for all three patients (j) and tumor-to-background ratios derived from the MFI data in all three patients (k). 


\section{Flowchart real-time surgical decision making}

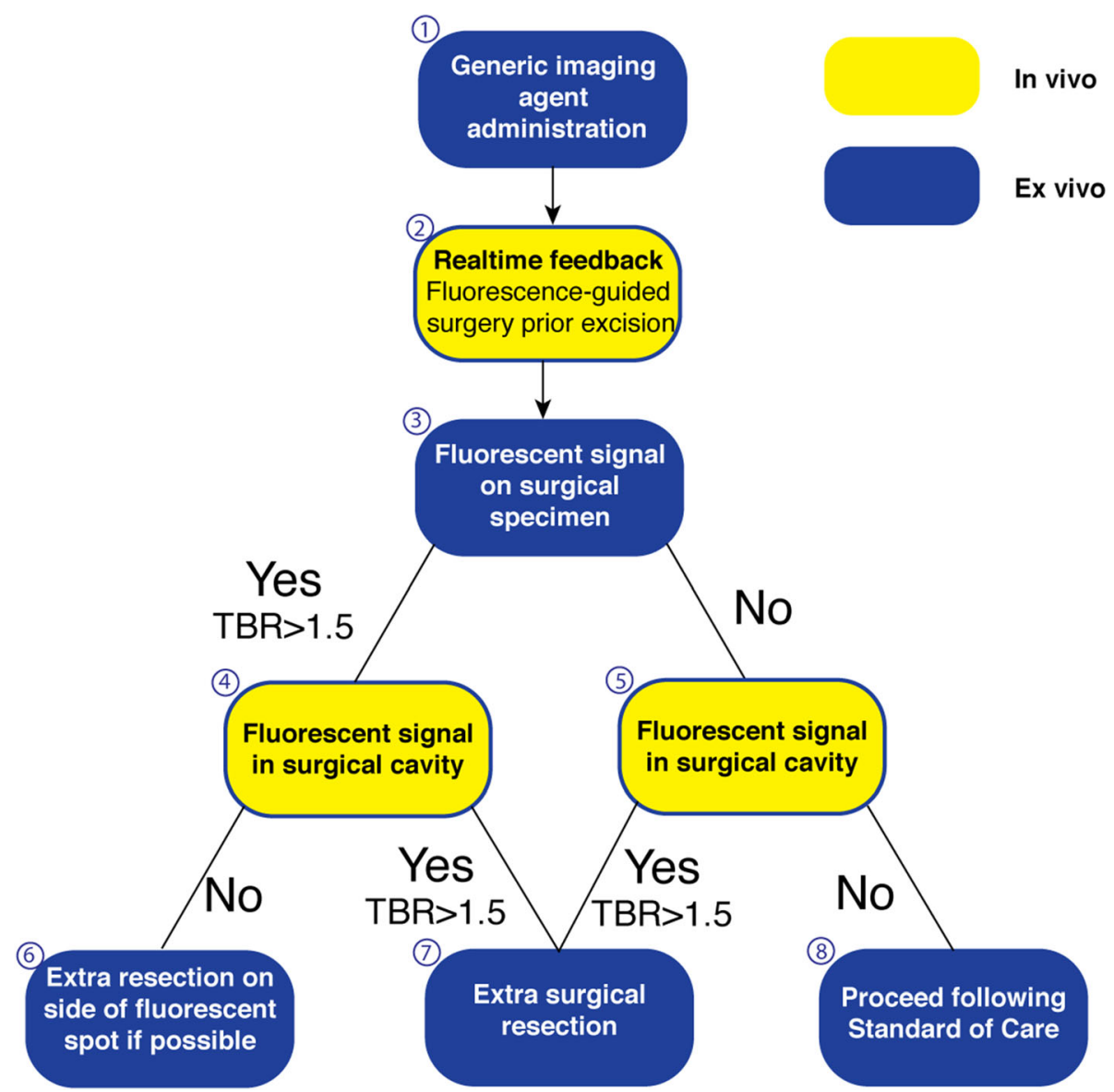

Fig. 4. Flowchart real-time clinical decision-making. Suggested flowchart for fluorescence-guided surgery and surgical decision-making using fluorescence with this tumor generic fluorescence imaging agent. An additional surgical resection can be made in different scenarios when fluorescent spots with a tumor-to-background ratio of $>1.5$ are observed on either the surgical specimen or in the surgical cavity.

accounts not only for head and neck cancer patients, the surgeon receives immediately feedback on the clinical situation and is able to alter the surgical plan (i.e., no further surgery, marking of positive spot for post-operative radiotherapy).

In the current study, intra-operative ex vivo fluorescence imaging for margin assessment has been prioritized above in vivo fluorescence imaging. Ex vivo specimen imaging using a closed-field and standardized imaging device allows for standardization of measurements, which is highly relevant in fluorescence-guided imaging where multiple factors like distance to the camera, angle of illumination, and environmental light can influence imaging results severely [14]. With the availability of a close-field imaging device at the surgical theatre, tissue can be imaged immediately after surgical excision allowing for direct surgical decision-making, as also has been described by other groups $[11,15,16]$. As the close-field imaging device used in the current study has a limited field of view, future developments in field of view, resolution, and imaging quality might further enhance intra-operative clinical decision-making.

Despite the investigated imaging agents showed promising results in previous studies, visual discrimination between tumor and non-tumor tissue remains challenging due to high, a specific background signals in non-tumor tissue $[5,7,8$, 12]. A low background signal, a consequence of the design of ONM-100, shows a clear and sharply delineated fluorescent signal in tumors in this study. This discriminative strength of ONM-100 is illustrated by the fact that the tumor-positive resection margins were clearly highlighted compared to the non-involved adjacent resection margin. Moreover, ONM-100 was administered within $24 \mathrm{~h}$ of surgery, providing major benefits over other fluorescence imaging agents which are administered $2-5$ days prior to surgery $[5,7,17]$. Indeed, we have seen some false-positive 
fluorescence signal in salivary gland tissue, as is previously described as well [5]. The exact reason for this activation needs to be further analyzed in future studies; however, as we believe that a surgeon is able to differentiate salivary gland tissue from tumor tissue, we believe this might not be a significant clinical problem.

Based on our experience as a tertiary referral hospital, performing complex surgery often in late-stage HNSCC disease (Supplemental Figure S3), we identified three possible scenarios originating from the findings in this study following the detection of a fluorescent lesion. First, fluorescent lesions can be resected with an adequate margin without compromising vital structures. Second, an identified fluorescent lesion cannot be resected due to anatomical borders or the extent of disease. Third, new unidentified lesions not directly related to the initial tumor or irresectable lesions are identified which might change or cease the surgical plan. The first and last scenario occurred in this particular study. As this study shows, the fluorescent lesions detected at the excised specimen correlated in $100 \%$ of the cases to tumor-positive resection margin and in $60 \%$ of the cases to close resection margins. This implicates that, in theory, in ten patients, an immediate re-resection could be considered, thereby preventing a second surgery. This is highly relevant, since not all patients were eligible for second surgery due to their intrinsic comorbidities. From the possible ten re-resections, one re-resection would result in a case of overtreatment due to a false-positive fluorescent signal according to the current guidelines on tumor involved margins. If this eventually would lead to a higher local tumor control remains to be studied. We assume that in case of a successful immediate re-resection at the primary tumor site, adjuvant therapy can at least be de-intensified from the concomitant combination of chemoradiation to adjuvant radiotherapy alone. We therefore consider these data encouraging for further exploring the use of ONM-100 for the purpose of intra-operative margin assessment in a larger phase II trial.

Recent studies in HNSCC suggest a cutoff point of $2 \mathrm{~mm}$ between a tumor-positive and a tumor-negative surgical margin, as $>2 \mathrm{~mm}$ margin shows no significant increase in disease-specific survival $[3,18]$. Therefore, a method that could discriminate between tumor presence within $2 \mathrm{~mm}$ and $>2 \mathrm{~mm}$ of the surgical border would be suited for real-time surgical guidance in HNSCC patients. Interestingly, all fluorescence-negative surgical margins found in this study had a surgical margin of at least $>2 \mathrm{~mm}$, again underlining the clinical applicability of this technique. It should be noted that the numbers of tumor-positive margins detected in the current study are outside the average norm [19]. However, a tertiary referral hospital (UMCG) receives delicate and latestage disease patients which a high a priori chance for tumor-positive surgical margins.

In conclusion, this study demonstrates the potential added value of specimen-driven fluorescence imaging of the surgical specimen in HNSCC patients after administration of the optical imaging agent ONM-100. We found a $100 \%$ sensitivity for tumor-positive surgical margin detection using this technique and showed the potential for the intraoperative detection of occult disease. ONM-100 is easy to implement into standard clinical care due to the clear visual discrimination between tumor and non-tumor tissue, therefore having great potential in assisting in clinical decisionmaking during HNSCC surgery, preventing under- and overtreatment.

Supplementary Information. The online version contains supplementary material available at https://doi.org/10.1007/s11307-021-01614-z.

Acknowledgements. We want to thank all patients and their families for participating in this study. We would like to thank the pathology department of the UMCG (Eric, Lilo, and Maaike) for their excellent assistance during pathology processing and physician assistant Rachel Dopheide for her help in recruiting study patients.

Author Contribution. PJS, FJV, BDS, and YJ designed the study. PJS and FJV performed data acquisition, analyzed and interpreted data, and drafted the manuscript. PJS and FJV contributed equally to this work. JG designed the polymer composition for ONM-100. BvdV and JJD were involved in histopathological analyses and reviewing of the manuscript. GMvD and MJHW designed and supervised the study, interpreted data, and drafted the manuscript. KPS, WK, SAHJdV, and MJHW performed surgery and fluorescence imaging procedures. All authors reviewed and critically revised the final manuscript.

\section{Declarations}

\section{Conflict of Interest}

BDS and JG are advisors of OncoNano Medicine Inc. BDS, JG, and YJ are shareholders of OncoNano Medicine Inc. GMvD is member of the Scientific Advisory Board of Exotome LLC and AxelaRx Biosciences Inc., CEO, founder, and shareholder of TRACER Europe BV.

Open Access This article is licensed under a Creative Commons Attribution 4.0 International License, which permits use, sharing, adaptation, distribution and reproduction in any medium or format, as long as you give appropriate credit to the original author(s) and the source, provide a link to the Creative Commons licence, and indicate if changes were made. The images or other third party material in this article are included in the article's Creative Commons licence, unless indicated otherwise in a credit line to the material. If material is not included in the article's Creative Commons licence and your intended use is not permitted by statutory regulation or exceeds the permitted use, you will need to obtain permission directly from the copyright holder. To view a copy of this licence, visit http:// creativecommons.org/licenses/by/4.0/.

\section{References}

1. Mitchell DA, Kanatas A, Murphy C, Chengot P, Smith AB, Ong TK (2018) Margins and survival in oral cancer. Br J Oral Maxillofac Surg 56:820-829. https://doi.org/10.1016/j.bjoms.2018.06.021

2. Wong LS, McMahon J, Devine J, McLellan D, Thompson E, Farrow A, Moos K, Ayoub A (2012) Influence of close resection margins on local recurrence and disease-specific survival in oral and oropharyngeal carcinoma. Br J Oral Maxillofac Surg 50:102-108. https:// doi.org/10.1016/j.bjoms.2011.05.008

3. Binahmed A, Nason RW, Abdoh AA (2007) The clinical significance of the positive surgical margin in oral cancer. Oral Oncol 43:780-784. https://doi.org/10.1016/j.oraloncology.2006.10.001 
4. van Keulen S, Nishio N, Fakurnejad S, Birkeland A, Martin BA, Lu G, Zhou Q, Chirita SU, Forouzanfar T, Colevas AD, van den Berg NS, Rosenthal EL (2019) The clinical application of fluorescenceguided surgery in head and neck cancer. J Nucl Med 60(6):758-763. https://doi.org/10.2967/jnumed.118.222810

5. Voskuil FJ, de Jongh SJ, Hooghiemstra WTR, Linssen MD, Steinkamp PJ, de Visscher SAHJ, Schepman KP, Elias SG, Meersma GJ, Jonker PKC, Doff JJ, Jorritsma-Smit A, Nagengast WB, van der Vegt B, Robinson DJ, van Dam GM, Witjes MJH (2020) Fluorescence-guided imaging for resection margin evaluation in head and neck cancer patients using cetuximab-800CW: a quantitative dose-escalation study. Theranostics 10:3994-4005. https://doi.org/ $10.7150 /$ thno. 43227

6. Voskuil FJ, Steinkamp PJ, Zhao T et al (2020) Exploiting metabolic acidosis in solid cancers using a tumor-agnostic pH-activatable nanoprobe for fluorescence-guided surgery. Nat Commun 11:32573210. https://doi.org/10.1038/s41467-020-16814-4

7. Rosenthal EL, Warram JM, de Boer E, Chung TK, Korb ML, Brandwein-Gensler M, Strong TV, Schmalbach CE, Morlandt AB, Agarwal G, Hartman YE, Carroll WR, Richman JS, Clemons LK, Nabell LM, Zinn KR (2015) Safety and tumor specificity of cetuximab-IRDye 800 for surgical navigation in head and neck cancer. Clin Cancer Res 21:3658-3666. https://doi.org/10.1158/10780432.CCR-14-3284

8. Gao RW, Teraphongphom NT, van den Berg NS, Martin BA, Oberhelman NJ, Divi V, Kaplan MJ, Hong SS, Lu G, Ertsey R, Tummers WSFJ, Gomez AJ, Holsinger FC, Kong CS, Colevas AD, Warram JM, Rosenthal EL (2018) Determination of tumor margins with surgical specimen mapping using near-infrared fluorescence. Cancer Res 78:5144-5154. https://doi.org/10.1158/0008-5472.CAN18-0878

9. Kossatz S, Brand C, Gutiontov S, Liu JTC, Lee NY, Gönen M, Weber WA, Reiner T (2016) Detection and delineation of oral cancer with a PARP1 targeted optical imaging agent. Sci Rep 6:21371. https:// doi.org/10.1038/srep21371

10. Voldborg BR, Damstrup L, Spang-Thomsen M, Poulsen HS (1997) Epidermal growth factor receptor (EGFR) and EGFR mutations, function and possible role in clinical trials. Ann Oncol 8:1197-1206. https://doi org/10.1023/A:1008209720526 "keywords":["phenotype”,"mutation”,"cancer”,"“cell
11. van Keulen S, Nishio N, Birkeland A, Fakurnejad S, Martin B, Forouzanfar T, Cunanan K, Colevas AD, S. van den Berg N, Rosenthal E (2019) The sentinel margin: intraoperative ex vivo specimen mapping using relative fluorescence intensity. Clin Cancer Res 25:4656-4662. https://doi.org/10.1158/1078-0432.CCR-19-0319

12. Zhao T, Huang G, Li Y, Yang S, Ramezani S, Lin Z, Wang Y, Ma X, Zeng Z, Luo M, de Boer E, Xie XJ, Thibodeaux J, Brekken RA, Sun X, Sumer BD, Gao J (2016) A transistor-like pH nanoprobe for tumour detection and image-guided surgery. Nat Biomed Eng 1:1546. https://doi.org/10.1038/s41551-016-0006

13. Tummers WS, Warram JM, van den Berg NS, Miller SE, Swijnenburg RJ, Vahrmeijer AL, Rosenthal EL (2018) Recommendations for reporting on emerging optical imaging agents to promote clinical approval. Theranostics 8:5336-5347. https://doi.org/10.7150/ thno. 27384

14. Koch M, Symvoulidis P, Ntziachristos V (2018) Tackling standardization in fluorescence molecular imaging. Nat Photonics 2018 12:9 12:505-515. https://doi.org/10.1038/s41566-018-0221-5

15. Fakurnejad S, Krishnan G, Van Keulen S (2020) Intraoperative molecular imaging for ex assessment of peripheral margin in oral squamous cell carcinoma. Front Oncol 1476. https://doi.org/10.3389/ fonc. 2019.01476

16. de Jongh SJ. Tjalma JJJ, Koller M. (2020) Back-table fluorescenceguided imaging for circumferential resection margin evaluation using bevacizumab-800CW in patients with locally advanced rectal cancer. J Nucl Med 61:655-661. https://doi.org/10.2967/jnumed.119.232355

17. Zhang RR, Schroeder AB, Grudzinski JJ, Rosenthal EL, Warram JM, Pinchuk AN, Eliceiri KW, Kuo JS, Weichert JP (2017) Beyond the margins: real-time detection of cancer using targeted fluorophores. Nat Rev Clin Oncol 14:347-364. https://doi.org/10.1038/ nrclinonc.2016.212

18. Zanoni DK, Migliacci JC, Xu B, Katabi N, Montero PH, Ganly I, Shah JP, Wong RJ, Ghossein RA, Patel SG (2017) A proposal to redefine close surgical margins in squamous cell carcinoma of the oral tongue. JAMA Otolaryngol Head Neck Surg 143:555-560. https:// doi.org/10.1001/jamaoto.2016.4238

19. Orosco RK, Tapia VJ, Califano JA, Clary B, Cohen EEW, Kane C, Lippman SM, Messer K, Molinolo A, Murphy JD, Pang J, Sacco A, Tringale KR, Wallace A, Nguyen QT (2018) Positive surgical margins in the 10 most common solid cancers. Sci Rep 8:5686. https://doi.org/ $10.1038 / \mathrm{s} 41598-018-23403-5$

Publisher's Note. Springer Nature remains neutral with regard to jurisdictional claims in published maps and institutional affiliations. 